\title{
DMRC Location Tracker and Alert System
}

\author{
Ruchika Jain ${ }^{1}$ | Anu Rathee ${ }^{2}$ \\ ${ }^{1}$ B.Tech Scholar, Information Technology, Maharaja Agrasen Institute of Technology, Rohini, Delhi \\ ${ }^{2}$ Assistant Professor, Information Technology, Maharaja Agrasen Institute of Technology, Rohini, Delhi
}

To Cite this Article

Ruchika Jain and Anu Rathee, "DMRC Location Tracker and Alert System”, International Journal for Modern Trends in Science and Technology, 6(12): 253-256, 2020.

\section{Article Info}

Received on 10-November-2020, Revised on 02-December-2020, Accepted on 06-December-2020, Published on 11-December-2020.

\section{ABSTRACT}

It is a simple React-Native application used to get an alert before your destined metro station so that you can plan your journey accordingly without the fear of missing the destination. The purpose of this project is to design and construct a hand-held wireless tracking device so that the location of a person can be detected. Other than providing alert the application can also be used for women safety purpose. It focuses on one of the important problems of society that is eve-teasing. It makes the society to be aware of the misbehavior which is happening and to come forward for help. It enables women to be self-dependent and can handle any kind of unpredictable situation. It mainly shares the location based on GPS, (Global Positioning System). Pressing the SOS button it sends the emergency message with time and location to the emergency contacts and metro authorities. When the application triggers on the time of travelling it send the location to the emergency contacts with few images taken by the camera on spot, and a siren rings which gives immediate help.

KEYWORDS: React native application, alert system, GPS based tracker

\section{INTRODUCTION}

Currently, there are many facilities provided to women in DMRC for making themselves independent.

It is really mandatory today for women to travel alone with ease, safety, and confidence. The best way to reduce chances in becoming a victim of violent crime (robbery, sexual assault, rape) is to identify and call on resources to help you out of unsafe situations. In this paper, we provide such facility so that women can travel alone and safe. Women not knowing much about travelling in DMRC will get a facility in which an alert will be given to them so that they won't miss their metro station, helping them to be confident in travelling. This application is designed in such a way to get you a help with minimal and unnoticeable efforts. This application sends the emergency message with time and location to the emergency contacts and metro authorities with few images taken by the camera on spot hence providing immediate help. This reduces risk and provides safety when needed.

As the project is made in React-Native environment using react.js and javascript, it focuses on the development of more projects using the web development environment. Most of the React-Native API's are cross platform and will work on platforms like iOS and Android. You can develop complete, full-blown applications that look, run and feel native without writing one line of platform-specific code. Moreover, React-Native is compatible with third-party plugins. To add Google Maps' functionality is simply downloaded as a third party plugin which is then linked with native modules. As the project focus on making women safe and independent, many other features can further be developed so that more and more women can travel DMRC alone and safe. 


\section{RELATED WORKS}

There are numerous works done related to location tracker and alert system using different algorithms in technical world.

\section{Android Based Safety Triggering Application [P.Kalyanchakravarthy]-}

This paper describes a few Safety Triggering applications being developed and its successful implementation with tested results. The application has target users those sections of the people that surprisingly fall under a situation where instant communication of their whereabouts becomes indispensable to be told to certain authorized persons at remote end. This application main purpose is for women's safety. When we feel that we are in emergency situation, for example travelling alone in the Auto/Cab at night time we can use this application so that on one click we can send our location to our family members and to any police stations continuously until we stop with password based button.

\section{An Android Application For Women Safety [Bramarambika Thota and Udaya Kanchana Kumar.P]}

The usage of smart phones equipped with GPS navigation unit have increased rapidly from 3\% to quite $20 \%$ within the past five years. Hence, a smart phone is often used efficiently for private safety or various other protection purposes especially for ladies. This paper presents Sauver, a private safety application developed for smart phones of android platform. This app are often activated by one click when the user feels she is in peril .This application communiqués the user's location to the registered contacts for each few seconds in the form of message. Thus, it acts sort of a sentinel following behind the person till the user feels.

\section{All in one Intelligent Safety System for Women Security [Abhijit Paradkar and Deepak Sharma] According to the reports of WHO,} NCRB-socialgovernment organization 35\%Women everywhere the planet face tons of unethical physical harassment publicly places like railway-bus stands, foot paths etc. In this paper the authors have reviewed of varied existing systems on women security. The authors have felt a requirement of advanced women security system to provides the security measure publicly places also as travelling alone through public transports (school buses, company vehicle etc). This paper proposed a replacement model for the ladies security publicly places which aims to supply the $100 \%$ safe environment.

\section{METHODOLOGY}

As stated in the objective this React-Native application provides an alert before destination and SOS messages in case of an emergency. In the beginning, we researched and analysed many related works and papers. We collected useful information regarding google maps, GPS, location tracking and sending SOS messages.

\section{[1] System Requirements}

- React Native

- Firebase

- Expo

- Google cloud Api's

- Android device

\section{[2] Flow of application}

The flow of the application goes in following way.

\section{Login into application}

The metro riders register into the application and provide their credentials. Once they are registered their data will be stored in the database and they can then login into the application.

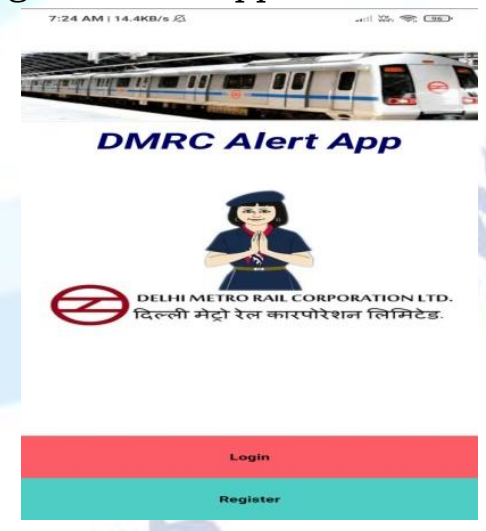

Fig: 1 Home Page

\section{Getting alert before destination}

The metro rider needs to fill their initial and final destination. The application will take the initial position of the rider using GPS of the android device (figure1) on which the application is running. With the detection of latitudinal and longitudinal coordinates of the location, the application will then find the exact location of the rider using Google maps. 
Clicking calculate button will then calculate transit distance, transit duration, total fare, from, to.

When the distance to the destination will be less than $400 \mathrm{~m}$ an alert will be provided to the rider. Also, distance left and time left will be upgraded at an interval of $1 \mathrm{~min}$.

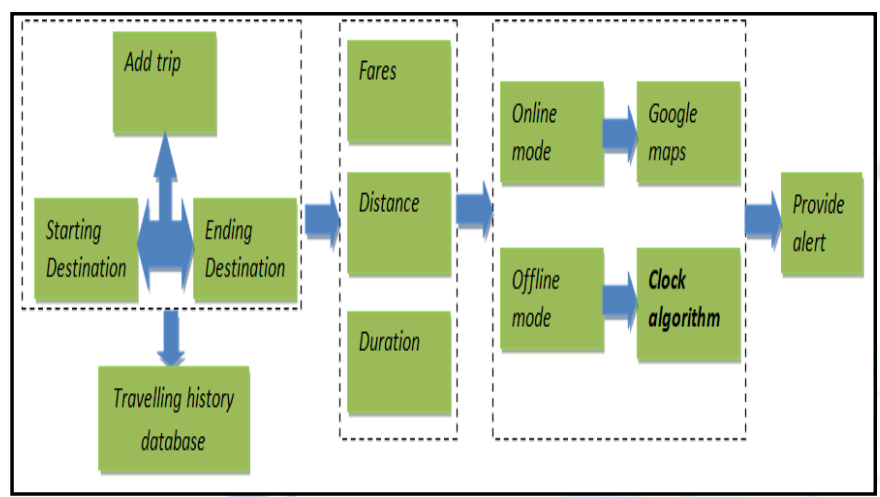

Fig: 2 Flowchart of how application provides alert.

\section{Sending SOS messages}

In emergencies, the SOS button will help in sending SOS messages to the Metro authorities and emergency contacts. This SOS message will contain a message informing that the rider is in danger with time and location, and few images taken by the camera on the spot. This system is also integrated with mobile speakers so that loud noise is produced to alert the people nearby.

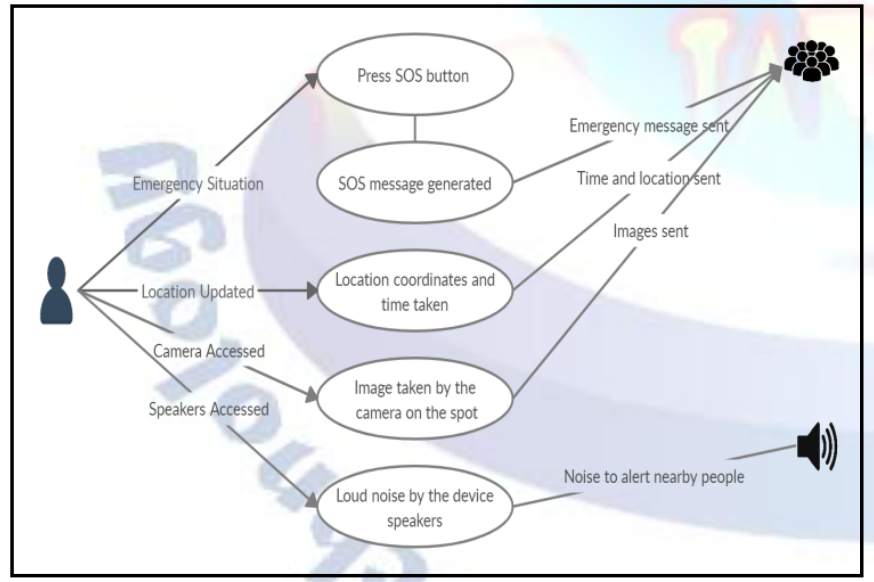

Fig: 3 Use case diagram of providing SOS messages

\section{RESULTS}

\section{[1] Getting Alert before destination}

- Initial result - Initially when origin and destination are filled the app calculates total distance and duration and displays it. (Fig: 4)

- Intermediate results- These results show distance left and time left. Distance left and time left changes every 1 min. (Fig:6)

- Final result- Final result shows an alert when 400 m left. (Fig:5)

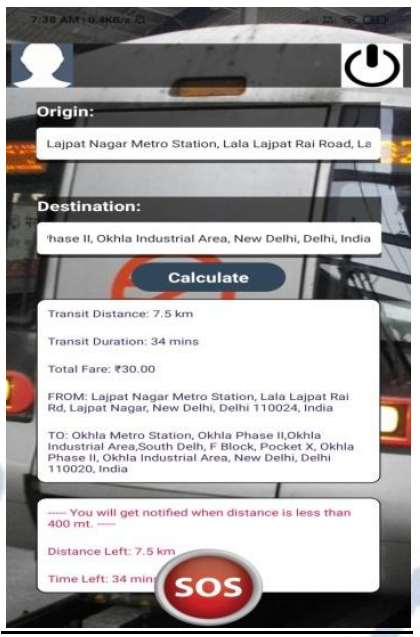

Fig: 4 Initial results

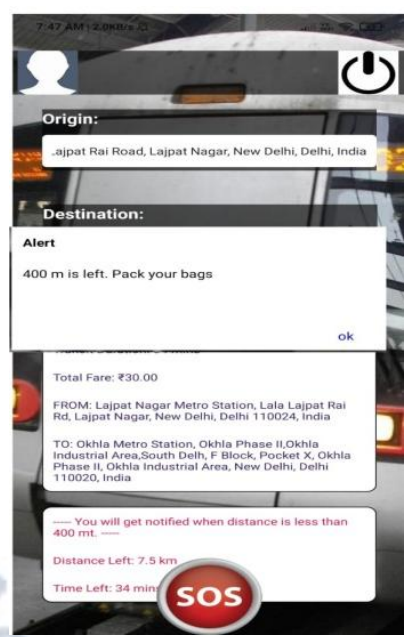

Fig: 5 Final results

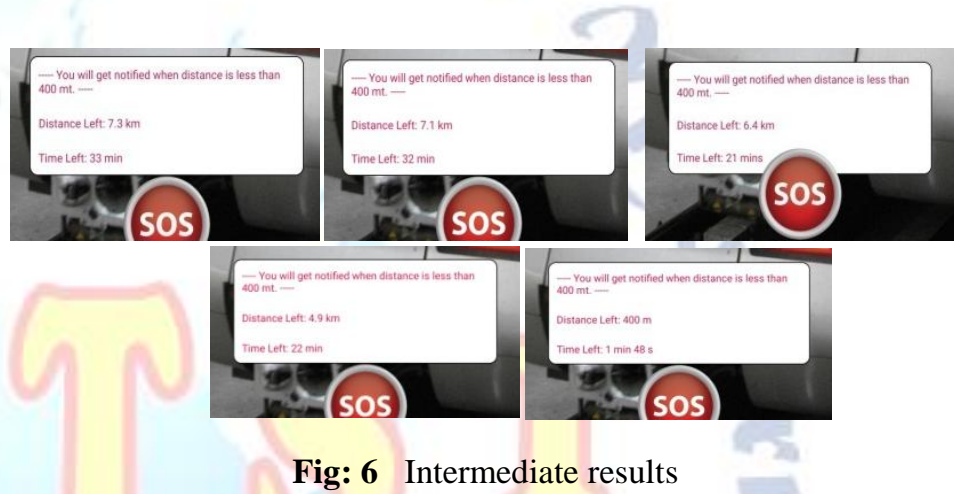

\section{[2] Sending SOS messages}

On clicking SOS button camera pops up and on again clicking SOS button picture is clicked by the camera (Fig:7) and sent to the emergency contacts (Fig:8) with location of the user and an emergency message (Fig:9).

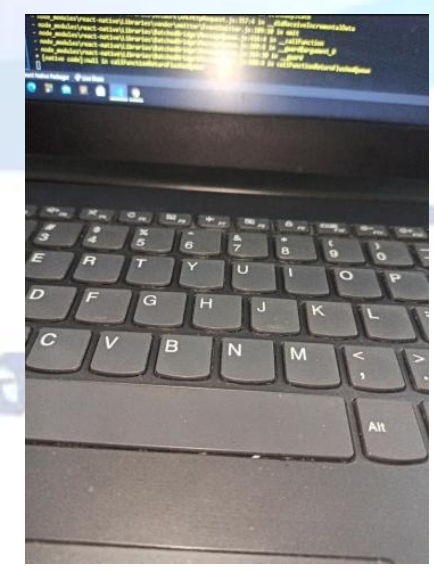

Fig: 7 Picture clicked

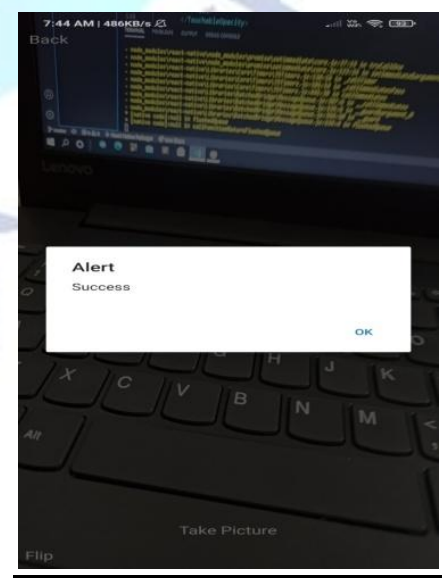

Fig: 8 Picture sent 
The Emergency message contains a sentence stating "Hi I am in emergency, Need help", location of the user and an image URL.

Message recipient after clicking on URL can see the picture clicked by the camera (Fig: 10).

The message will be received by three recipients (Fig: 11).
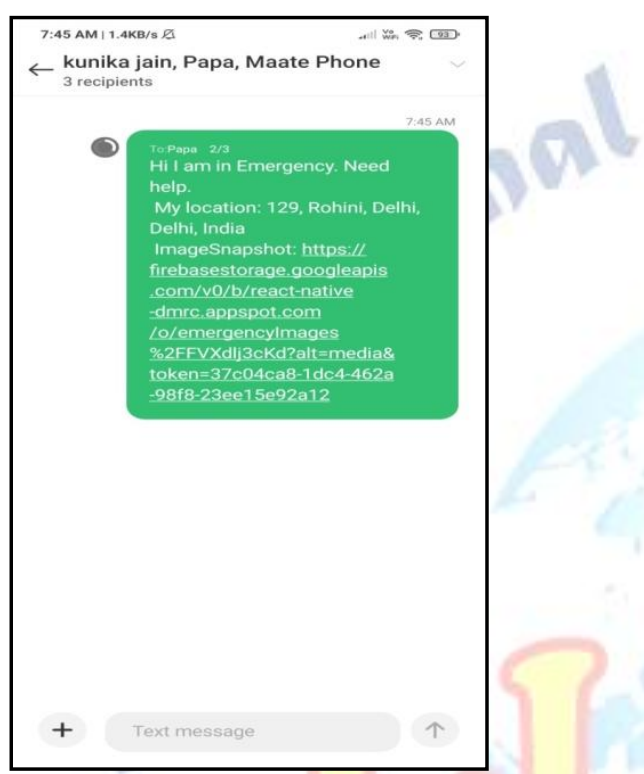

Fig: 9 Message delivered successfully

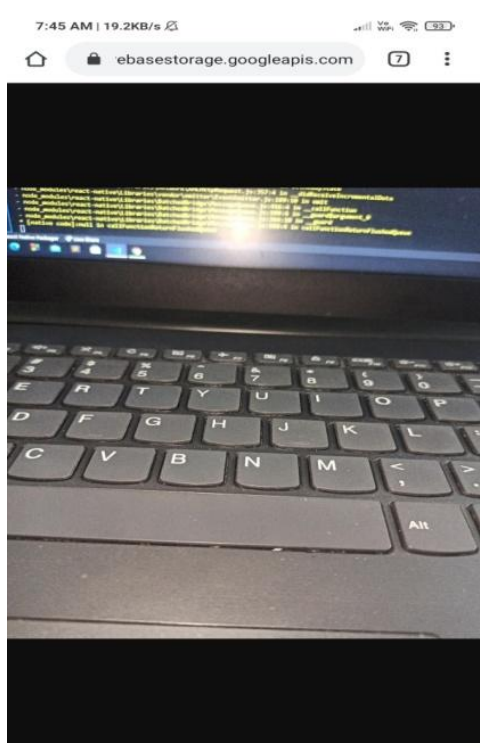

Fig: 10 Image after clicking on image URL

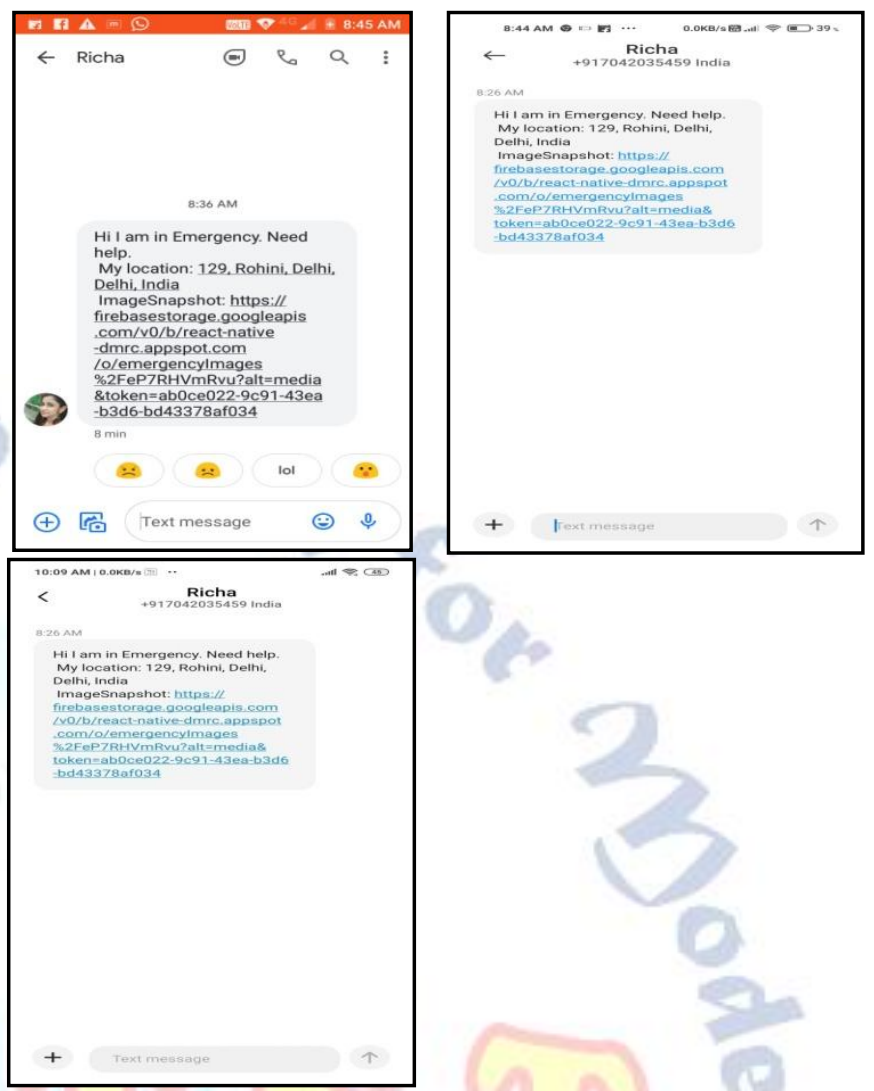

Fig: 11 Message received successfully

\section{v. CONCLUSION}

In this project a react native application is made to run on Android platforms. The application will provide an update of distance left and time left for their destination after every one minute and provides the alert before $400 \mathrm{~m}$. The SOS messages are sent to the emergency contacts with few images. We had worked on the application and got accurate results.

\section{REFERENCES}

[1] P.Kalyanchakravarthy1, T.Lakshmi2, R.Rupavathi2, S.Krishnadilip2, P.Lakshmankumar2" Android Based Safety Triggering Application" International Journal of Computer Science and Information Technologies, Vol. 5 (1), 2014, 646-647

[2] Bramarambika Thota, Udaya Kanchana Kumar .P," Sauver: An Android Application For Women Safety" International Journal Of Technology Enhancements And Emerging Engineering Research, Vol 3, Issue 05

[3] Abhijit Paradkar and Deepak Sharma, "All in one Intelligent Safety System for Women Security" International Journal of Computer Applications (0975 - 8887) Volume 130 No.11, November2015 